\title{
Antifungal Resistance Among Candida Species From Patients with Genitourinary Tract Infection at Muhammad Abdullahi Wase Specialist Hospital, Kano - Nigeria.
}

\author{
${ }^{*}$ D.W. Taura, ${ }^{1}$ M.H. Maje, ${ }^{2}$ A.M. Koki, and ${ }^{2 M}$.G. Musa \\ 1Department of Microbiology, Bayero University, P.M.B. 3011, Kano - Nigeria \\ 2Department of Medical Microbiology, Abdullahi Wase Specialist Hospital, Kano- Nigeria \\ [*Corresponding author: Email ; tauradw@yahoo.com. 梁: +2348036267381]
}

\begin{abstract}
The increasing incidence of Candidiasis affecting the genitourinary tracts as well as the introduction of new antifungal drugs has recently highlighted the need for performing fungal susceptibility tests. To determine the antifungal resistance among Candida species from the genitourinary tracts, 689 Urine and High vaginal swab (HVS) samples were collected from female patients clinically diagnosed with genitourinary tract infection between September 2011 to January 2012. The samples were inoculated onto Sabouraud dextrose agar (SDA). Isolates from SDA were placed on Corn Med agar (CMA) to ensure detection of mixed cultures. Germ tube tests were performed for identification of the isolates. Susceptibility tests were carried on the isolates using broth dilution method. The occurrence rate of Candida species were as follows: Candida albicans 124 (48.4\%), Candida glabrata 89 (34.8\%), Candida krusei 23 (9.0\%) and Candida Tropicalis 20 (7.8\%). The rate of occurrence of Candida species in high vaginal swab $76(61.3 \%)$ was significantly higher than that of urine $48(38.7 \%)$. Distribution of Candida species among different age groups show that, the highest incidence was in age brackets 20 - $30158(61.7 \%)$, while that of $41-50$ and above $8(3.1 \%)$ had the least. High rate of susceptibility was observed for each isolate against Fluconazole $23(65.7 \%)$ and Ketoconazole $22(62.9 \%)$. The resistance rate was 12 (34.3\%) for Fluconazole and Ketoconazole $13(37.1 \%)$. These results incriminate $C$. albicansas the most common Candida species causing genitourinary tract infection in women. This surveillance study has established Fluconazole and Ketoconazole as very effective antifungal agents for the treatment of genitourinary tract infections caused by Candida species.
\end{abstract}

Keywords: Antifungal resistance, Candida species, Genitourinary tract and Infections.

\section{INTRODUCTION}

Candida species are opportunistic yeast affecting the genitourinary tracts. It belongs to the subclass Ascomycota and measures $2-4 \mathrm{~mm}$ in diameter (Prescott et al., 2008). The genus Candida consists of more than 160 species. Candida can variously be found among humans, other mammals, birds, insects, arthropods, fish, animal waste, plants, mushrooms, honey, nectar, fresh water, sea water and in the air (Moosaet al., 2004). Candida is listed by the Center for Disease Control (CDC) as a cause of sexually transmitted disease (Prescott et al., 2008). No other mycotic pathogen produces as diverse a spectrum of opportunistic disease in humans as does Candida. Candida species are important nosocomial pathogens and can be transmitted sexually (Tatfeng et al., 2004). Candida species are the normal micro biota within the gastrointestinal tracts, respiratory tracts, vaginal area and the mouth (Prescott et al., 2008). Candidiasis refers to a range of infection caused by species of fungal genus Candida. The infections can be acute or chronic, localized or systemic. Disseminated Candidiasis is frequently life threatening. The great majority of these infections are caused by Candida albicans (Greenwood et al., 1992). Pathogenic Candida infections mainly occur as opportunistic infections due to altered conditions of the host, and during the fungus proliferates faster (Elegbe et al., 1982). The incidence of genitourinary tract infection is much higher in females during adolescence and childbearing years (Sobel, 2004). Candida is found in the vagina of $35-50 \%$ of healthy women. Under some conditions, such as reduced immunity, prolonged antibiotics therapy, use of contraceptives, malnutrition, pregnancy, diabetes, obesity, tissue transplant, use of immunesuppression drugs (Corticosteroids), neutropenia, Candida may become pathogenic and cause Candidiasis (Okungbowa et al., 2003). Candida species are the second most frequent isolates from blood cultures in hospitals with large populations of immunocompromised patients (Beck Sagueet al., 1993; Tatfeng et al., 2004). Vulval pruritis is the dominant feature of vulvovaginal Candidiasis. Women may complain of dysuria, soreness, irritation, dyspareunia, suprapubic pains, and haematuria, white and clumpy vaginal discharge. The discharge is classically described as thick, adherent, and "cottage cheese-like" with a pH of 4.0-4.5 (Tatfenget al., 2004). The diagnosis is confirmed by finding the organism on a wet mount of the discharge. Microscopy may be negative in up to $50 \%$ of patients 
with confirmed genitourinary Candidiasis (Sobel et al., 2004). The many drugs that are available at present to treat fungal infections can be divided into four broad groups on the basis of their mechanism of actions. These antifungal agents inhibit macromolecule synthesis (Flucytosine), impair membrane barrier function (polyenes), inhibit ergosterol synthesis (allylamines, thiocarbamates, azole derivatives, and morpholines) or interact with microtubules (Vanden et al., 1997). Currently, the azole drugs comprising Miconazole, Ketoconazole, Fluconazole and Itraconazole are widely used for the treatment of fungal infections. Emergence of drug resistance among yeast isolates and consequent increase in serious fungal infections have been reported (DeMuri et al., 1995). The mechanisms of resistance to these antifungal agents by yeast isolates are purely chromosomal as Candida species lack plasmid or other natural mechanisms capable of transferring genetic materials between strains (Odds et al., 2003). Knowledge of mechanisms of antifungal resistance has been valuable in identifying resistant isolates and using them to validate in vitro measurement systems (VandenBossche et al., 1998; White et al., 1998; Ghannoum et al., 1999; Moore et al., 2000). This paper aimed to determine the antifungal resistance of Candida species among female patients clinically diagnosed with genitourinary tract infections with special emphasis on the wellstudied National Committee for Clinical Laboratory Standards (NCCLS, 2002) methodologies.

\section{MATERIALS AND METHODS}

\section{Sample collection}

All the samples were transported to the Microbiology Laboratory of the Muhammad Abdullahi Wase Specialist Hospital (MAWSH) in Kano-Nigeria for analysis. A total of 689 patients recruited for the study between September, 2011 to January, 2012. The samples comprised 449 high vaginal swabs (HVS) and Endo Cervical Swabs (ECS) and 240 samples of Urine collected from women clinically diagnosed for genitourinary tract infections and swab sticks were used for the collection of HVS while urine samples were collected using sterile universal containers.

\section{Ethical consideration}

Ethical Clearance was obtained from the Hospital Management Board, Kano-Nigeria.

\section{Processing of urine samples}

The urine sample was poured into clean, dry sterile plastic test tube and then centrifuged at a speed of 3000rpm for 5 minutes. After centrifugation, the supernatant was decreased. A little fluid remaining was used to reconstitute the sediment in the tube. An inoculating wire loop calibrated to hold $0.01 \mathrm{ml}$ of urine was sterilized using Bunsen flame. The loop was allowed to cool down. The loop was inserted vertically into the urine sediment inside the test tube. A loopful of urine sediment was applied to a small area of the Sabouraud Dextrose Agar (Bio-Mark, India) plate to make a pool. The inoculating loop was then used to spread the inoculums from the pool.

A wet film was prepared by placing a drop of the urine sediment on a clean dry glass slide. The slide was then covered with clean cover slips and then examined under a microscope for yeast cells using the 10X objective lens. The 40X objective lens was used to confirm the presence or absence of yeasts and other infectious agents.

\section{Processing of High Vaginal Swabs (HVS) and Endo-Cervical Swabs (ECV)}

The mouth of the tube containing the swab was passed rapidly through the flame of a Bunsen burner to make the mouth area sterile. The swab was removed from the tube and then applied to a small area of Sabouraud dextrose agar (SDA) (Bio-Mark, India) plate to make a pool.

A few drops of normal saline were added to the swab in the tube and the mixture was shaken to dislodge materials from the swab into the saline. The swab was withdrawn and a sterile inoculating loop was used to transfer and spread a small amount of the saline onto a clean dry slide to make a smear. The smear was allowed to air-dry. The dried smear was stained by Gram's Method. The smear was then examined under the microscope for yeast cells using the oil immersion objective lens.

A wet film was prepared by placing a drop of the saline from the tube onto a clean dry glass slide. The slide was covered with cover slip and then examined under the microscope for yeast cells and pseudohyphae first using the 10X objective before switching to 40X objective lens to confirm the presence or absence of yeasts (Chessbrough, 2004)

\section{Culture procedure}

Samples were cultured on Sabouraud Dextrose Agar (SDA) at $37^{\circ} \mathrm{C}$. Inoculated plates were examined after $48 \mathrm{~h}$ incubation. Isolates from SDA were placed on Corn Meal agar (C M A) to ensure detection of mixed cultures. Cultures were incubated at $37^{\circ} \mathrm{C}$ for $72 \mathrm{~h}$. Identification of Candida species were based on colony morphology and appearance of athroconidia, 
pseudohyphae and Blastoconidia on the C- M agar (chessbrough, 2004)

\section{Germ Tube Test (GTT)}

All yeasts isolated from the specimens processed were identified to the species level using the germ tube test and Dalamus Test for Morphological features of the Specimens.

Using Pasteur pipette, three drops of fresh human serum (obtained from the serology laboratory at the MAWSH) were dispensed into labeled test tubes. Using a sterile inoculating loop, a colony of yeasts was transferred into the serum in the labeled test tubes. The colony was emulsified in the serum. The set up was incubated at $37^{\circ} \mathrm{C}$ for about 3 hours. Using a Pasteur pipette, a drop of the suspension taken from the test tube after incubation was placed on a clean dry slide. The suspension was covered with a clean cover glass. The slide was examined under a microscope for germ tubes on the yeasts using the 10X objective lens. A germ tube is a tubelike outgrowth that arises from the yeast cell. The 40X objective was used to confirm the presence or absence of germ tubes. When yeasts with germ tubes were seen, the culture was reported as Candida albicans isolated. When the yeast cells do not show germ tubes, the culture was reported as other Candida Spp isolate.

\section{RESULTS}

Out of a total of 689 (HVS/ECS and Urine) samples collected, two hundred and fifty six(256) yielded growth of yeast isolates as shown in Figure 1.These were identified as C. albicans 124 (48.4\%), Candida glabrata 89 (34.8\%),C. krusei $23(9.0 \%)$ and C. tropicalis 20 (7.8\%). A comparison of the occurrence of these species in high vaginal swabs (HVS) \& Endo Cervical Swabs (ECS) and Urine showed that, their rate of occurrence was significantly higher in HVS/ECS (66.4\%) than in Urine (33.6\%) $(\mathrm{P} \leq 0.05)$.
On the distribution of Candida species among different age groups, revealed that age group 21-30 had the highest frequency distribution of Candida species with a total of $158(61.7 \%)$ which indicates highest percentage prevalence of Candida species while age group between 41-50 recorded the lowest frequency with $8(3.1 \%)$ as in shown in Figure 2.

Tables 1 and 2 indicated the summaries of the Minimum Inhibitory Concentration (MIC) of the isolates against different concentrations (0.1$100 \mathrm{\mu g} / \mathrm{ml}$ ) of Fluconazole and Ketoconazole with reference to NCCLS standard, isolates given clarity of growth (optical clarity) at concentration $\leq 10 \mu \mathrm{g} / \mathrm{ml}$ were regarded as susceptible while those giving such clarity at concentrations $>10 \mu \mathrm{g} / \mathrm{ml}$ were regarded as resistant. From the results,23(65.7\%) and 22 (62.9\%) Candida albicans isolates gave optical clarity at lower concentrations $(\leq 10 \mu \mathrm{g} / \mathrm{ml})$ and were regarded as susceptible to Fluconazole and Ketoconazole respectively. The remaining 12 (34.3\%) and13 (37.1\%) C. albicans isolates which gave optical clarity at higher concentrations $(>10 \mu \mathrm{g} / \mathrm{ml})$ were regarded as resistant to Fluconazole and Ketoconazole respectively. Similarly, 29(82.9\%) and 19(54.3\%) C. glabrata isolates gave optical clarity at lower concentrations $(\leq 10 \mu \mathrm{g} / \mathrm{ml})$ and were regarded as susceptible to Fluconazole and Ketoconazole respectively. While 6(17.1\%) and 16(45.7\%) Candida glabrata isolates which gave optical clarity at higher concentrations (>10 $\mathrm{gg} / \mathrm{ml})$ were regarded as resistant to Fluconazole and Ketoconazole, respectively. There was no growth with the regards to Candida krusei and C. tropicalis. With respect to antifungal Fluconazole and Ketoconazole show high activity of $72 \%$ and $62 \%$ respectively against C. albicans isolates while their resistance rates of $45 \%$ and $54 \%$ respectively were however moderate. There was no significant difference $(P \geq 0.05)$ between the activities of both drugs. These observations were further summarized in Figures 1 and 2 and Tables 1 to 3 .

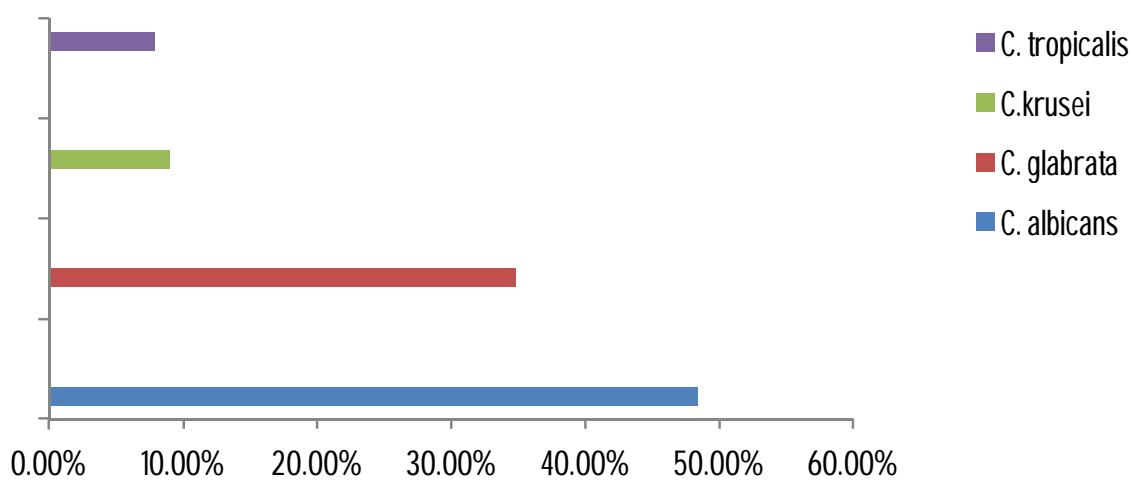

Figure 1: Occurrence of Candida species in the genitourinary tract. 


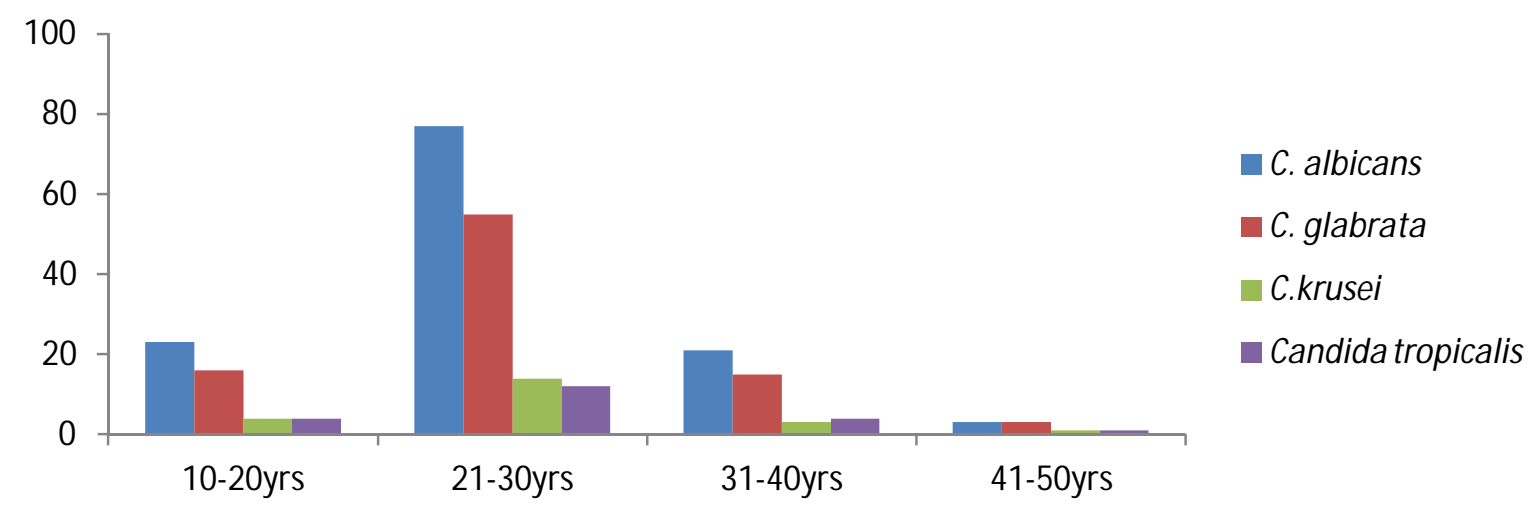

Fig. 2 Distribution of Candidiasis within different age groups.

Table 1: Susceptibility of Candida Spp to Fluconazole

\begin{tabular}{ccccccccc}
\hline $\begin{array}{c}\text { Candida } \\
\text { Spp }\end{array}$ & $\begin{array}{c}0.25 \mu \mathrm{g} / \\
\mathrm{ml}\end{array}$ & $\begin{array}{c}0.5 \\
\mu \mathrm{g} / \mathrm{ml}\end{array}$ & $\begin{array}{c}1.0 \\
\mu \mathrm{g} / \mathrm{ml}\end{array}$ & $\begin{array}{c}5.0 \\
\mu \mathrm{g} / \mathrm{ml}\end{array}$ & $\begin{array}{c}10.0 \\
\mu \mathrm{g} / \mathrm{ml}\end{array}$ & $\begin{array}{c}50.0 \\
\mu \mathrm{g} / \mathrm{ml}\end{array}$ & $\begin{array}{c}100 \\
\mu \mathrm{g} / \mathrm{ml}\end{array}$ & $\begin{array}{c}\mathrm{MIC} \\
\mu \mathrm{g} / \mathrm{ml}\end{array}$ \\
\hline C.albicans & $R$ & $R$ & $S$ & $S$ & $S$ & $S$ & $S$ & 1.0 \\
C.albicans & $R$ & $S$ & $S$ & $S$ & $S$ & $S$ & $S$ & 0.5 \\
C.albicans & $R$ & $R$ & $R$ & $S$ & $S$ & $S$ & $S$ & 5.0 \\
C.albicans & $R$ & $R$ & $R$ & $R$ & $S$ & $S$ & $S$ & 10.0 \\
C.albicans & $R$ & $R$ & $S$ & $S$ & $S$ & $S$ & $S$ & 1.0 \\
C. glabrata & $R$ & $S$ & $S$ & $S$ & $S$ & $S$ & $S$ & 0.5 \\
C. glabrata & $R$ & $R$ & $S$ & $S$ & $S$ & $S$ & $S$ & 1.0 \\
C. glabrata & $R$ & $R$ & $S$ & $S$ & $S$ & $S$ & $S$ & 1.0 \\
C. glabrata & $R$ & $R$ & $S$ & $S$ & $S$ & $S$ & $S$ & 1.0 \\
C. glabrata & $R$ & $R$ & $R$ & $S$ & $S$ & $S$ & $S$ & 5.0
\end{tabular}

MIC= Minimal inhibitory Concentration $\mathbf{R}=$ Resistance $\mathbf{S}=$ Susceptible

NOTE: The MIC was regarded as the lowest antifungal concentration with substantially lower turbidity compared to growth in the antifungal free growth control well

Table 2: Susceptibility of Candida Spp to Ketoconazole

\begin{tabular}{ccccccccc}
\hline $\begin{array}{c}\text { Candida } \\
\text { Spp }\end{array}$ & $0.25 \mu \mathrm{g} / \mathrm{ml}$ & $\begin{array}{c}0.5 \\
\mu \mathrm{g} / \mathrm{ml}\end{array}$ & $1.0 \mu \mathrm{g} / \mathrm{ml}$ & $5.0 \mu \mathrm{g} / \mathrm{ml}$ & $\begin{array}{c}10.0 \\
\mu \mathrm{g} / \mathrm{ml}\end{array}$ & $\begin{array}{c}50.0 \\
\mu \mathrm{g} / \mathrm{ml}\end{array}$ & $\begin{array}{c}100 \\
\mu \mathrm{g} / \mathrm{ml}\end{array}$ & $\begin{array}{c}\mathrm{MIC} \\
\mu \mathrm{g} / \mathrm{ml}\end{array}$ \\
\hline C. albicans & $R$ & $R$ & $R$ & $S$ & $S$ & $S$ & $S$ & 5.0 \\
C. albicans & $R$ & $R$ & $R$ & $R$ & $S$ & $S$ & $S$ & 10.0 \\
C. albicans & $R$ & $R$ & $S$ & $S$ & $S$ & $S$ & $S$ & 1.0 \\
C. albicans & $R$ & $R$ & $S$ & $S$ & $S$ & $S$ & $S$ & 1.0 \\
C. albicans & $R$ & $R$ & $S$ & $S$ & $S$ & $S$ & $S$ & 1.0 \\
C. glabrata & $R$ & $R$ & $R$ & $S$ & $S$ & $S$ & $S$ & 5.0 \\
C. glabrata & $R$ & $R$ & $R$ & $S$ & $S$ & $S$ & $S$ & 5.0 \\
C. glabrata & $R$ & $R$ & $R$ & $S$ & $S$ & $S$ & $S$ & 5.0 \\
C. glabrata & $R$ & $R$ & $R$ & $R$ & $S$ & $S$ & $S$ & 10.0 \\
C. glabrata & $R$ & $R$ & $R$ & $S$ & $S$ & $S$ & $S$ & 5.0 \\
\hline MIC= Minimal inhibitory Concentration & $\mathrm{R}=$ Resistance S = Susceptible & & & &
\end{tabular}

Table 3. Susceptibility of isolates to Fluconazole and Ketoconazole.

\begin{tabular}{lcccc}
\hline \multicolumn{1}{c}{ Isolate } & No. R (\%) & No.S (\%) & No. R (\%) & No. S (\%) \\
\hline Candida albicans & $22(17.7)$ & $40(32.3)$ & $24(19.4)$ & $38(30.6)$ \\
Candida glabrata & $8(9)$ & $38(42.7)$ & $20(22.5)$ & $23(25.8)$ \\
Candida krusei & Nil & Nil & Nil & Nil \\
Candida Tropicalis & Nil & Nil & Nil & Nil \\
Total & $30(14.1)$ & $78(36.6)$ & $44(20.7)$ & $61(28.6)$ \\
\hline
\end{tabular}

$\mathbf{R}=$ Resistance $\mathbf{S}=$ Sensitive $\quad$ Nil $=$ No Organism was tested at this Concentration 


\section{DISCUSSION}

This study identified the particular species of yeast that causes infections among patients that attended Abdullahi Wase Specialist Hospital. The study also determined the in vitro susceptibility of the yeast species isolated from various clinical samples. The results reveal that, Candida albicans had the highest incidence rate of $48.4 \%$ among the yeast isolates studied which is in line with the reports of Akorthaet al, (2009) who reported the incidence rate of $63.9 \%$ for Candida albicans. Kurtzmanet al, (2006) reported the prevalence rate of $76 \%$ forCandida albicans among the yeast isolates studied. Sobelet al, (1995) reported $80-90 \%$ incidence rate of Candida albicans. Similarly, Tatfenget al, (2003) reported C. albicans to be the most incriminated yeast isolate in the urinary tract infection. These findings however, contradict the earlier report of Okungbowet al (2003) who reported $C$. glabrata as the most common Candida species among the symptomatic individual in Nigerian Cities. However, the present results show that the overall frequency distribution of Candida $S p$ $(61.7 \%)$ was found to be higher among $21-30$ years age bracket which is in conformity with the work of many researchers. These include Sehgal, (1990) who reported the incidence rate of $54 \%$ within the age bracket of 20-30 in Northern Nigeria. Akorthaet al, (2009) reported the incidence rate of $57 \%$ within the age range of 26-36 in Edo/ Benin City. Okungbowa et al. (2003) reported the incidence rate of $35 \%$ within the agerange of $25-35 \%$ in Benin City. This work point to this age range as the most vulnerable age group. This may be due to the use of contraceptives, drug abuse or even due to sexual promiscuity. MIC determination was employed for the further fungal because it is often perceived as the ideal method of antimicrobial drugs susceptibility testing. The antifungal agents used in this study were chosen on the basis of being commonly available on the local market and were among the drugs that are prescribed at the Abdullahi Wase Specialist Hospital. The Fluconazole susceptibility rate of $65.7 \%$ observed is however lower than the report of Akorthaet al, (2009) who reported 95.7\% susceptibility rate for Fluconazole. The resistance rate of $34.3 \%$ recorded for a resistance rate of $3.6 \%$ for candida albicans is higher than the report of by Sobel et a.I, 2004. In another study, no Fluconazole resistance was reported among yeast isolates in US, England and Brazil, (Sobel, et al 2004), (Dodgson, et al, 2004) and (Cohen, et al 2002).

\section{CONCLUSION}

The present study incriminated $C$. albicansas the most common Candida Spp causing genitourinary tract infections. Ketoconazole and Fluconazole are the most active antifungal agents for the treatments of genitourinary tract infections in the study area.

\section{REFERENCES}

Akortha, E.E., Nwaugo ,V.O., and Chikwe, N.O. (2009). Antifungal resistance among Candida species from patients with genitourinary tract infection isolated in Benin City. African Journal of Microbiology Research, 3(11): 694-699.

Beck-Sagué, C.M. and Jarvis, W.R. (1993). Secular trends in the epidemiology of nosocomial fungal infections in the United States, 19801990. National Nosocomial Infections Surveillance. The Journal of Infectious Diseases, 167(5):1247-1251.

Cohen, L.E., Anderson, J.B. and Kohn, L.M. (2002). Evaluation of drug resistance in Candida albicans, Annual Review of Microbiology, 56: 139-165.

DeMuri, G.P, and Hostetter, M.K. (1995). Resistance to antifungal agents. Pediatric Clinics of North America, 42: 66585.

Dodgson, A.R., Dodgson, K.J., Pujol, C., Pfaller, M.A. and Soll, D.R.(2004): Clade specific flucytosine resistance is due to a single nucleotide change in the FURI gene of candida albicans. Antimicrobial Agents and Chemotherapy, 48(6): 2223-2227.

Elegbe IA, Bofu M (1992). Direct microscopical versus cultural method in screening for candidiasis among gravid Nigerian women. Mycopathology 79: $137-139$.

Ghannoum, M. A. and Rice, L. B. (1999) Antifungal agents: mode of action, mechanisms of resistance and correlation of these mechanisms with bacterial resistance. Clinical Microbiology Review, 12 (4): 501-517.

Greenwood, D., Slack, R.C.B. and Pentherer, J.F. (1992). Medical microbiology. A guide to microbial infections: pathogenesis, immunity, laboratory diagnosis and control. 14th ed. Churchhill Livingstone, London.

Jawetz EJ, Melmic L, Adelberg EA (2001). Medical microbiology.22 ${ }^{\text {nd }}$ ed. Lange books/McGraw Hill, London.p. 1017.

Kurtzman, C.P. and Fell, J.W. (2006).Yeast systematics and phylogeny- implications of molecular identification methods for studies in ecology. Biodiversity and Ecophysiology of Yeasts, The yeast handbook, Springer. 
Moore, C.B., Sayers, N., Mosquera, J., Slaven, J. and Denning, D.W. (2000). Antifungal drug resistance in Aspergillus. Journal of Infection, 41: 203-220.

Moosa, M.Y., Sobel, J.D., Elhalis, H., Du, W. and Akins, R.A. (2004). Fungicidal activity of fluconazole against Candida albicansin a synthetic vagina-simulative medium. Antimicrobial Agents and Chemotherapy, 48(1): 161-7.

National Committee for Clinical Laboratory Standard (2002).Reference method for Broth dilution antifungal susceptibility testing of yeasts. Approved standard M27-A2, 2nd ed. National Committee for Clinical Laboratory Standards, Wayne, Pa. Segal, B.H., Kwon-Chung, J., Walsh, T.J., Klein, B. S., Batiwalla, M., Almyroudi, N.G., Holland, S.M. and Romani, L. (2006). Immunotherapy for fungal infections. Clinical Infectious Diseases, 42: 507-515

Odds FC, Brown, A.J.P. and Gow, N.A.R. (2003). Antifungal agent: mechanisms of action. Trends in Microbiology, 6: 276 - 279.

Okungbowa, F.I., Isuehuemhen OS, Dede A (2003).The distribution frequency of Candida species in the genitourinary tract among symptomatic individuals in Nigeria cities. Revista Iberoamericana de Micología, 20: 6063.
Prescott JP, Harley JM, Klein DA (2008). Microbiology, 7th ed. McGraw Hill publication. New York USA.

Sobel JD, Wiesenteld HC, Martens M, Danna P, Hooton IM, Rompalo A, Sperling M, Livengood IIIC, Horowitz B, Thron JV, Edwards L, Panzer $H$, Chu TC (2004). Maintenance fluconazole therapy for recurrent vulvovaginal candidiasis, New England Journal of Medicine, 351: 876883.

Tatfeng YM, Agba Ml, Nwobu GO, Agbonlahor DE (2004). Candida albicans in urinary tract or seminal sac. Online Journal of Health and Allied Sciences, 2(4): 4-5.

Vanden BH, Marichal P, Odds FC (1997). Mechanisms of antifungal resistance. Trends in Microbiology, 14: 44 - 49.

Vanden-Bossche, H., Dromer, F., Improvisi, I., Lozano-Chiu, M., Rex, J.H. and Sanglard, D. (1998). Antifungal drug resistance in pathogenic fungi. Medical Mycology, 36: 119128.

White, T.C., Marr, K.A. and Bowden, R.A. (1998). Clinical, cellular, and molecular factors that contribute to antifungal drug resistance. Clinical Microbiology Review, 11: 382-402. 